\title{
Representação spinorial de variedades SpinC em formas espaciais
}

\author{
Samuel Augusto Wainer ${ }^{1}$ \\ Instituto Tecnológico de Aeronáutica \\ Rafael de Freitas Leão ${ }^{2}$ \\ Insitituto de Matemática, Estatística e Computação Científica
}

Resumo. Dois tópicos de pesquisa bem conhecidos no século XX, na área de geometria diferencial, são a equação de Dirac e imersões minimais de superfícies. Em 1998, Thomas Friedrich, elucidou a relação entre imersões isométricas de superfícies com uma dada curvatura média e soluções da equação de Dirac. Na literatura, outros autores abordaram o problema da relação entre soluções da equação de Dirac e imersões isométricas para outras variedades Spin em espaços de dimensões maiores. Aqui enunciamos a caracterização spinorial de imersões isométricas de variedades que possuem uma estrutura $\operatorname{Spin}^{\mathbb{C}}$ de dimensão arbitrária em formas espaciais.

Palavras-chave. Álgebra de Clifford, Spinores, Imersões.

\section{Spinores e imersões}

Em 1998, Thomas Friedrich [4], utilizando o formalismo do fibrado de Clifford, elucidou a relação entre imersões isométricas de superfícies com uma dada curvatura média $H$ e soluções da equação de Dirac.

A ideia principal de Friedrich, que leva à descrição de uma superfície $M^{2}$ por um campo spinorial, é a observação de que a restrição para $M^{2}$ de qualquer campo spinorial paralelo $\psi$ em $\mathbb{R}^{3}$ é um campo spinorial não-trivial $\varphi$ em $M^{2}$ de norma constante que é uma solução da equação de Dirac

$$
D \varphi=H \varphi .
$$

Por outro lado, qualquer solução $\varphi$ da equação $D \varphi=H \varphi$ de norma constante define um endomorfismo simétrico $E: T\left(M^{2}\right) \rightarrow T\left(M^{2}\right)$ tal que o campo do spinor satisfaz uma equação tipo Killing

$$
\nabla_{X} \varphi=E(X) \cdot \varphi
$$

\footnotetext{
${ }^{1}$ wainer@ita.br

${ }^{2}$ leao@ime.unicamp.br.
} 
As condições de integrabilidade resultantes para o endomorfismo $E$ são exatamente as equações de Gauss e Codazzi, isto é, uma solução $\varphi$ da equação de Dirac produz uma imersão isométrica de $M^{2}$ em $\mathbb{R}^{3}$.

Especificamente, o teorema provado por Friedrich em [4] é

Teorema 1.1. Seja $\left(M^{2}, g\right)$ uma variedade Riemanniana 2-dimensional orientada e $H$ : $M^{2} \rightarrow \mathbb{R}$ uma aplicação suave. Então são equivalentes as seguintes afirmações:

(a) Existe uma imersão isométrica $\left(\tilde{M}^{2}, g\right) \rightarrow \mathbb{R}^{3}$ do recobrimento universal de $M^{2}\left(\tilde{M}^{2}\right)$ em $\mathbb{R}^{3}$ com curvatura média $H$.

(b) Existe uma solução $\varphi$, de norma constante $|\varphi|=1$, da equação de Dirac $D \varphi=H \varphi$.

(c) Existe um par $(\varphi, E)$ consistindo de um endomorfismo simé trico E tal que $\operatorname{tr}(E)=$ - H e um campo spinorial $\varphi$ tal que $\nabla_{X}^{M^{2}} \varphi=E(X) \cdot \varphi$.

Na literatura, outros autores abordaram o problema da relação entre soluções da equação de Dirac e imersões isométricas para outras variedades em espaços de dimensões maiores:

1. Em 2004, Bertrand Morel, [9] estendeu a representação spinorial de imersões isométricas em $\mathbb{R}^{3} 3$ para $S^{3}$ e $H^{3}$. Seu argumento, bem como Friedrich, consiste em considerar spinores especiais em $S^{3}$ e $H^{3}$ e suas restrições às superfícies imersas.

2. Em 2008 Marie-Amelie Lawn [5] mostrou como uma dada superfície de Lorentziana $\left(M^{2}, g\right)$ pode ser imerso no espaço pseudo-riemanniano $\mathbb{R}^{2,1}$.

3. Em 2010 Lawn e Julien Roth [6] forneceu uma caracterização spinorial de superfícies imersas isometricamente em formas espaciais 4-dimensional e espaços de produto $\mathbb{M}^{3} \times \mathbb{R}\left(\mathbb{M}^{3} \simeq\left(\mathbb{R}^{3}, \mathbb{S}^{3}, \mathbb{H}^{3}\right)\right.$

4. Lawn e Roth [7] em 2011, deram um caracterização spinorial de superfícies imersas isometricamente de assinatura arbitrária em formas espaciais pseudo-riemannianas tridimensionais. Isso generaliza o trabalho de Lawn em $\mathbb{R}^{2,1}$ a outras formas espaciais de Loretzianas.

5. Em 2013 Bayard [1] provou que uma imersão de uma superfície Riemanniana $M^{2}$ no espaço de Minkowski quadridimensional $\mathbb{R}^{1,3}$, com um fibrado normal dado $E$ e dado um vetor de curvatura média $\vec{H} \in \Gamma(E)$, é equivalente a um campo spinorial normalizado $\varphi \in \Gamma(\Sigma E \otimes \Sigma M)$ solução de uma equação de $\operatorname{Dirac} D \varphi=\vec{H} \cdot \varphi$ na superfície.

6. Recentemente, no ano de 2017, Bayard, Lawn e Roth [2] estudaram a representação spinorial de subvariedades de qualquer dimensão e qualquer codimensão em formas espaciais em termos da existência de spinores de Killing generalizados. A ideia dos autores deste artigo, que inspirou nosso trabalho, é considerar a representação na álgebra de Clifford $C l_{n}$ dada pela multiplicação da esquerda. Essa representação não é irredutível, mas tem vantagens algébricas muito interessantes. 
7. E, finalmente em 2017 Bayard, Roth e Jimenez [3] apresentaram essa caracterização spinorial de subvariedades, de qualquer dimensão e codimensão, em grupos de Lie equipados com uma métrica invariante à esquerda.

\section{Representação Spinorial de variedades SpinC em formas espaciais}

Todos esses resultados, se restringem a supor que os objetos em questão admitem uma estrutura Spin. Seguindo a ideia destes resultados recentes e utilizando um fibrado $\operatorname{Spin}^{\mathbb{C}}$ Clifford particular, nós apresentamos em [8] a representação spinorial de subvariedades $\operatorname{Spin}^{\mathbb{C}}$ de qualquer dimensão e codimensão em $\mathbb{R}^{n}$, estendendo-a para uma classe maior de variedades, como por exemplo variedades quase-complexas que admitem uma estrutura $\operatorname{Spin}^{\mathbb{C}}$ canônica.

Precisamente foi mostrado o seguinte teorema:

Teorema 2.1. Seja $M$ uma variedade Riemanniana p-dimensional, $E \rightarrow M$ um fibrado vetorial sobre $\mathbb{R}$ de posto $q$, assuma que TM e E são orientadas e $\operatorname{Spin}^{\mathbb{C}}(n=p+q)$. Suponha que $B: T M \times T M \rightarrow N$ é uma aplicação simétrica e bilinear. A é a conexão no fibrado $S^{1}$-principal. Assim as seguintes afirmações são equivalentes:

1. Existe um campo spinorial $\varphi$ tal que

$$
\nabla_{X} \varphi=-\frac{1}{2} \sum_{i=1}^{n} e_{i} \cdot B\left(X, e_{i}\right) \cdot \varphi+\frac{1}{2} i A(X) \cdot \varphi, \quad \forall X \in T M .
$$

2. Existe uma imersão isométrica $F: M \rightarrow \mathbb{R}^{n}$ com fibrado normal $E$ e segunda forma fundamental $B$.

Além disso, a 1-forma definida pelo produto interno

$$
\xi(X):=\langle\langle X \cdot \varphi, \varphi\rangle\rangle, \quad \forall X \in T M .
$$

é $\mathbb{R}^{n}$-valorada, fechada e $d F=\xi$.

Para o caso da representação spinorial de variedades Spin $^{\mathbb{C}}$ nos espaços de curvatura constante apresentamos as seguintes:

Teorema 2.2. Seja $M$ uma variedade $p$-dimensional, $E \rightarrow M$ um fibrado vetorial de posto q, assuma que TM e E são orientados e $\operatorname{Spin}^{\mathbb{C}}(n=p+q)$. Suponha que $B: T M \times T M \rightarrow$ $N$ é uma aplicação simétrica e bilinear. A é a conexão no fibrado $S^{1}$-principal, $\nu$ é o vetor normal de $\mathbb{S}^{n}$ em $\mathbb{R}^{n+1}$. As seguintes afirmações são equivalentes:

1. Existe um campo spinorial $\varphi$ tal que

$$
\nabla_{X} \varphi=-\frac{1}{2} \sum_{i=1}^{p} e_{i} \cdot B\left(X, e_{i}\right) \cdot \varphi+\frac{1}{2} X \cdot \nu \cdot \varphi+\frac{1}{2} \mathbf{i} A(X) \cdot \varphi, \quad \forall X \in T M .
$$


2. Existe uma imersão isométrica $F: M \rightarrow \mathbb{S}^{n}$ com fibrado normal E e segunda forma fundamental $B$.

Além disso, $F=\langle\langle\nu \cdot \varphi, \varphi\rangle\rangle \in \mathbb{S}^{n} \subset \mathbb{R}^{n+1}$.

Teorema 2.3. Seja $M$ uma variedade $p$-dimensional, $E \rightarrow M$ um fibrado vetorial de posto q, assuma que TM e E são orientados e $\operatorname{Spin}^{\mathbb{C}}(n=p+q)$. Suponha que $B: T M \times T M \rightarrow$ $N$ é uma aplicação simétrica e bilinear. A é a conexão no fibrado $S^{1}$-principal, $\nu$ é o vetor normal de $\mathbb{H}^{n}$ em $\mathbb{R}^{n, 1}$. As seguintes afirmações são equivalentes:

1. Existe um campo spinorial $\varphi$ tal que

$$
\nabla_{X} \varphi=-\frac{1}{2} \sum_{i=1}^{p} e_{i} \cdot B\left(X, e_{i}\right) \cdot \varphi-\frac{1}{2} X \cdot \nu \cdot \varphi+\frac{1}{2} \mathbf{i} A(X) \cdot \varphi, \quad \forall X \in T M .
$$

2. Existe uma imersão isométrica $F: M \rightarrow \mathbb{H}^{n}$ com fibrado normal $E$ e segunda forma fundamental $B$.

Além disso, $F=\langle\langle\nu \cdot \varphi, \varphi\rangle\rangle \in \mathbb{H}^{n} \subset \mathbb{R}^{n, 1}$.

\section{Conclusões}

É natural nos perguntarmos se a formulação apresentada em [2] pode ser adaptada para o caso de variedades $\operatorname{Spin}^{\mathbb{C}}$, por exemplo, no contexto de variedades quase-complexas a estrutura canônica que aparece é a estrutura $\operatorname{Spin}^{\mathbb{C}}$. Seguindo essa direção, os teoremas 2.1, 2.2 e 2.3 apresentam a representação spinorial de variedades $S_{p i n} \mathbb{C}$ em formas espaciais.

\section{Referências}

[1] P. Bayard, M. A. Lawn, J. Roth, Spinorial representation of surfaces in fourdimensional Space Forms, Annals of Global Analysis and Geometry, 44, pp. 433-453, 2013.

[2] P. Bayard, M. A. Lawn, J. Roth, Spinorial representation of submanifolds in Riemannian space forms, Pacific Journal of Mathematics, 291-1, pp. 51-80, 2017.

[3] P. Bayard, J. Roth, B. Z. Jimenez, Spinorial representation of submanifolds in metric Lie groups, Journal of Geometry and Physics 114, pp. 348-374, 2017.

[4] T. Friedrich, On the Spinor Representation of Surfaces in Euclidean 3-space, Jour. of Geom. and Phys. 28, pp. 143-157, 1998.

[5] M. A. Lawn, Immersions of Lorentzian surfaces in $\mathbb{R}^{2,1}$, Jour. of Geom. and Phys 58, pp. 683-700, 2008. 
[6] M. A. Lawn, J. Roth, Isometric immersions of hypersurfaces in 4-dimensional manifolds via spinors, Differential Geometry and its Applications, 28, pp. 205-219, 2010.

[7] M. A. Lawn, J. Roth, Spinorial Characterizations of Surfaces into 3-dimensional Pseudo-Riemannian Space Forms, Math. Physics, Analysis and Geometry, 14, pp. 185-195, 2011.

[8] R. F. Leão, S. A. Wainer, Immersion in $\mathbb{R}^{n}$ by complex spinors, Adv. Appl. Clifford Algebras (2018) 28: 44.

[9] B. Morel, Surfaces in $S^{3}$ and $H^{3}$ via spinors, Séminaire de Théorie spectrale et géométrie (Grenoble), 23, pp. 131-144, 2004-2005. 